\title{
Author Index Volume 8 (1997)
}

Abate, M. \& Patrizio, G., Kähler Finsler manifolds of constant holomorphic curvature

Alekseevsky, D.V. \& Marchiafava, $S$.,

Quaternionic transformations of a non-positive quaternionic Kähler manifold

Apanasov, B. \& Xie, X., Geometrically finite complex hyperbolic manifolds

Apostolov, V. \& Gauduchon, P., The Riemannian GoldbergSachs theorem

Archbold, R.J., Somerset, D.W.B., Kaniuth, E. \& Schlichting, G., Ideal spaces of the Haagerup tensor product of $C^{*}$-algebras

Aure, A., Decker, W., Hulek, K., Popescu, S. \& Ranestad, K., Syzygies of abelian and bielliptic surfaces in $\mathrm{P}^{4}$

Baird, P. \& Ou, Y.-L., Harmonic maps and morphisms from multilinear normpreserving mappings

Bauer, I., Irrational pencils on non-compact algebraic manifolds

Bezdek, K., Isoperimetric inequalities and the dodecahedral conjecture

Boyer, C.P. \& Galicki, K., The twistor space of a 3 Sasakian manifold

Branson, T. \& Hijazi, O., Vanishing theorems and eigenvalue estimates in Riemannian spin geometry

Bruno, A. \& Matsuki, K., Log Sarkisov program

Cerri, C., Non-commutative deformations of $C\left(\mathrm{~T}^{2}\right)$ and $K$-theory

Chen, J., Structures of certain Harmonic maps into Kähler manifolds

Chi, Q.-S., Merkulov, S. \& Schwachhöfer, L., Exotic holonomies $\mathrm{E}^{(a)}$

Dancer, A. \& Swann, A., The geometry of singular quaternionic Kähler quotients

Decker, W., see Aure
3 (1997) 301

6 (1997) 703

4 (1997) 421

1 (1997) 1

7 (1997) 849

2 (1997) 187

4 (1997) 441

$6(1997) 759$

1 (1997) 31

7 (1997) 921

4 (1997) 451

5 (1997) 555

5 (1997) 573

5 (1997) 583

5 (1997) 595

7 (1997) 849
Falbel, E., Gorodski, C. \&

Rumin, M., Holonomy of sub-Riemannian manifolds

Galicki, K., see Boyer

Gastesi, P.A., On Teichmïller spaces of Koebe groups

Gauduchon, P., see Apostolov

Gorodski, C., see Falbel

Guan, D.Z.-D., Toward a classification of almost homogeneous manifolds I - linearization of the singular extremal rays

Gudmundsson, S.,

Harmonic morphisms as sphere bundles over compact Riemann surfaces

Heffernan, D. \& Power, S.C., Limits of sums of digraph algebras with reduced digraphs having two vertices

Hijazi, $\mathbf{O}$. see Branson

Hoste, J. \& Przytycki, J.H., Tangle surgeries which preserve Jones-type polynomials

Hu, Y. \& Li, W.-P., Birational models of the moduli spaces of stable vector bundles over curves

Hulek, K. see Aure

Ionescu, P. \& Toma, M., On very ample vector bundles on curves

Izumi, $\mathrm{H}$.,

Constructions of non-commutative $L^{\mathbf{P}}$-spaces with a complex parameter arising from modular actions

Kalka, M., Mann, E., Yang, D. \&

Zinger, A., The exponential decay rate of the lower bound for the first eigenvalue of compact manifolds

Kaniuth, E., see Archbold

Kawamata, $Y$. On the cone of divisors of Calabi-Yau fiber spaces

Kraus, J. \& Ruan, Z.-J., Multipliers of Kac algebras
3 (1997) 317

1 (1997) 31

5 (1997) 611

4 (1997) 421

3 (1997) 317

8 (1997) 999

7 (1997) 935

1 (1997) 61

7 (1997) 921

8 (1997) 1015

6 (1997) 781

7 (1997) 849

5 (1997) 633

8 (1997) 1029

3 (1997) 345

1 (1997) 1

5 (1997) 665

2 (1997) 213 
Kurose, H. \& Nakagami, Y., Compact Hopf *-algebras, quantum enveloping algebras and dual Woronowicz algebras for quantum Lorentz groups

Kustermans, J. \& van Daele, A., $C^{*}$-algebraic quantum groups arising from algebraic quantum groups

Li, W.-P.,

see $\mathrm{Hu}$

Loi, P.H.

Periodic and strongly free automorphisms on inclusions of type III, factors

Loubeau, E., Pseudo harmonic morphisms

Mann, E.,. see Kalka

Marchiafava, S., see Alekseevsky

Masuda, T., An analogue of Longo's canonical endomorphism for bimodule theory and its application to asymptotic inclusions

Matsuki, K. \& Wentworth, R., Mumford-Thaddeus principle on the moduli space of vector bundles on an algebraic surface

Matsuki, K., see Bruno

Matsumoto, $\mathrm{K}$,

On $C^{*}$-algebras associated with subshifts

Mehta, V.B. \& Parameswaran,

A.J.,

On Wahl's conjecture for the Grassmannians in positive characteristic

Merkulov, S., see $\mathrm{Chi}$

Meyer, $\mathbf{R}$.

The Caratheodory pseudodistance and positive linear operators

Mingo, J.A. \& Nica, A., Crossings of set-partitions and addition of graded-independent random variables

Mochizuki, S.,

A version of the Grothendieck conjecture for $p$-adic local fields

Nagisa, M.,

Stable rank of some full group $C^{*}$-algebras of groups obtained by the free product

Nakagami, Y., see Kurose

7 (1997) 959
8 (1997) 1067
6 (1997) 781
1 (1997) 83
7 (1997) 943
3 (1997) 345
3 (1997) 301
$2(1997) 249$

2 (1997) 249

1 (1997) 97

4 (1997) 451

3 (1997) 357

4 (1997) 495

5 (1997) 583

$6(1997) 809$

$5(1997) 645$

4 (1997) 499

3 (1997) 375

7 (1997) 959
Nica, A.

see Mingo

Ou, Y.-L.,

see Baird

Parameswaran, A.J. see Mehta

Patrizio, G., see Abate

Pauly, C.

On pryms, rank 2 bundles and nonabelian theta functions

Perera, F.,

The structure of positive elements for $C^{*}$-algebras with real rank zero

Popescu, S., see Aure

Power, S.C., see Heffeman

Przytycki, J.H., see Hoste

Ranestad, K., see Aure

Ruan, Z.-J., see Kraus

Rumin, M., see Falbel

Sato, N.,

Two subfactors arising from a non-degenerate commuting square II - tensor categories and TQFT's

Schlichting, G., see Archbold

Schwachhöfer, L., see Chi

Seade, J. \& Suwa, T.,

Residues and topological invariants of singular holomorphic foliations

Shimomura, S.,

A system associated with the confluent hypergeometric function $F_{3}$ and a certain linear ordinary differential equation with two irregular singular points

Somerset, D.W.B., see Archbold

Starkov, A.N., Parabolic fixed points of Kleinian groups and the horospherical foliation on hyperbolic manifolds

Suwa, T., see Seade

Swann, A., see Dancer

Teleman, A. Non-abelian Seiberg-Witten theory and stable oriented pairs
5 (1997) 645

2 (1997) 187

4 (1997) 495

2 (1997) 169

2 (1997) 267

3 (1997) 383

7 (1997) 849

$1(1997) 61$

8 (1997) 1015

7 (1997) 849

2 (1997) 213

3 (1997) 317

3 (1997) 407

1 (1997) 1

5 (1997) 583

6 (1997) 825

5 (1997) 689

1 (1997) 1

2 (1997) 289

6 (1997) 825

5 (1997) 595

4 (1997) 507 
Toma, M., see Ionescu

Vaintrob, A., Melvin-Morton conjecture and primitive Feynman diagrams van Daele, A., see Kustermans

Wentworth, R., see Matsuki

$5(1997) 633$
$4(1997) 537$
$8(1997) 1067$
$1(1997) 97$

Winkelmann, J., Semicontinuity results for the topology of Taut manifolds $\mathrm{Xie}, \mathrm{X}$. see Apanasov

Yang, D., see Kalka

Zinger, A., see Kalka
1 (1997) 149 6 (1997) 703 3 (1997) 345 3 (1997) 345 